\title{
Combined subthalamic and nucleus basalis of Meynert deep brain stimulation for Parkinson's disease with dementia (DEMPARK-DBS): protocol of a randomized, sham-controlled trial
}

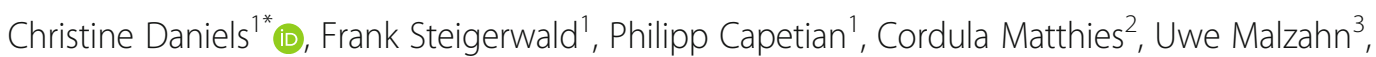

Peter U. Heuschmann ${ }^{3,4}$ and Jens Volkmann ${ }^{1}$

\begin{abstract}
Introduction: Dementia in Parkinson's disease (PDD) is a common non-motor symptom of advanced disease, associated with pronounced neocortical cholinergic deficits due to neurodegeneration of the nucleus basalis of Meynert (NBM) and its cholinergic terminals. In advanced PD, patients often require advanced therapies such as infusion therapy or deep brain stimulation (DBS) to improve motor control. However, patients with associated dementia are commonly excluded from DBS because of potential deterioration of cognitive functions. Yet marked reductions in dopaminergic medication and the subsequent risk of side effects (e.g., cognitive decline, psychosis, delirium) suggest that critical re-consideration of DBS of the subthalamic nucleus (STN-DBS) for advanced stages of PD and PDD is worthwhile. In this Phase $1 \mathrm{~b}$ study, we will provide STN-DBS to a cohort of PDD patients with severe motor fluctuations and combine two additional electrodes for augmentative neurostimulation of the NBM.

Methods: We aim to include 12 patients with mild-to-moderately severe PDD who fulfill indication criteria regarding motor symptoms for STN-DBS. Eligible patients will undergo implantation of a neurostimulation system with bilateral electrodes in both the STN and NBM. After 12 weeks of STN-DBS (visit 1N1), participants will be randomized to receive either effective neurostimulation of the NBM (group 1) or sham stimulation of the NBM (group 2). NBM-DBS will be activated in all participants after 24 weeks of blinded treatment (visit 2N2). The primary outcome will be the safety of combined bilateral STN- and NBM-DBS, determined by spontaneously-reported adverse events. Other outcome measures will comprise changes on scales evaluating cognition, activities of daily living functioning and clinical global impression, as well as motor functions, mood, behavior, caregiver burden and health economic aspects, and several domain-specific cognitive tests. Changes in scores (V1 - V2) for both treatment arms will undergo analysis of covariances, with baseline scores as covariates.

(Continued on next page)
\end{abstract}

\footnotetext{
* Correspondence: daniels_c@ukw.de

'Department of Neurology, University Hospital Würzburg, Josef-Schneider-Str.

11, 97080 Würzburg, Germany

Full list of author information is available at the end of the article
}

(c) The Author(s). 2020 Open Access This article is licensed under a Creative Commons Attribution 4.0 International License, which permits use, sharing, adaptation, distribution and reproduction in any medium or format, as long as you give appropriate credit to the original author(s) and the source, provide a link to the Creative Commons licence, and indicate if changes were made. The images or other third party material in this article are included in the article's Creative Commons licence, unless indicated otherwise in a credit line to the material. If material is not included in the article's Creative Commons licence and your intended use is not permitted by statutory regulation or exceeds the permitted use, you will need to obtain permission directly from the copyright holder. To view a copy of this licence, visit http://creativecommons.org/licenses/by/4.0/. 
(Continued from previous page)

Perspective: The feasibility and safety of combined STN-NBM-DBS in patients with PDD will be assessed to determine whether additional NBM-DBS improves or slows the progression of cognitive decline. Positive results would provide a basic concept for future studies evaluating the efficacy of NBM-DBS in larger PDD cohorts. Indirectly, proof-of-safety of STN-DBS in PDD might influence patient selection for this standard treatment option in advanced PD.

Trial registration: ClinicalTrials.gov identifier (NCT number): NCT02589925.

Keywords: Deep brain stimulation, Nucleus basalis of Meynert, Parkinson's disease, Parkinson's disease dementia, Subthalamic nucleus

\section{Introduction}

Cognitive impairment is a common non-motor symptom of Parkinson's disease (PD), and time of onset, severity, and cognitive profile show considerable interindividual variability [22]. Prevalence is high, with $80 \%$ of PD patients reported to experience dementia after 8 years [1]. Diagnosis of PD dementia (PDD) requires identification of cognitive deficits in at least two of the four core domains (attention, memory, executive and visuo-spatial functions) and consecutive impairment of normal functioning in everyday life [13].

Based on longitudinal neuropsychological examinations, Robbins and collaborators suggested a dual cognitive syndrome in PD: frontal-executive impairment and posterior cortical impairment [35, 40]. Frontal-executive functions (including flexibility, planning, switching between well-learned tasks, response inhibition, and working memory) may be impaired early in PD [10], and show some dopamine dependency [25]. Degeneration of the nigrostriatal and mesocorticolimbic dopamine pathways is supposed to be major pathophysiological correlate [25]. However, in some PD patients temporo-parietal cortical dysfunction (including impairment of visuospatial skills, semantic verbal fluency, auditory verbal learning, semantic and visual memory) complement or predominate the cognitive profile [2]. In non-demented patients with minimal cognitive impairment (MCI), visuospatial dysfunction is associated with a high risk of subsequent dementia [39]. Pagonabarraga et al. systematically evaluated a new neuropsychological test battery, with items sensitive for either frontal-executive or posterior cortical functions, and found that "cortical-type" item scores selectively helped to discriminate between demented and non-demented PD patients [32].

Progressive loss of cholinergic output from the nucleus basalis of Meynert (NBM) to neocortical regions may contribute to posterior cortical cognitive impairment [22]. In addition to degeneration of the dopaminergic system, severe loss of cholinergic neurons in the NBM is a consistent finding in neuropathological studies, and has been reported to differentiate PDD from nondemented PD patients [17]. Hall et al. performed an elaborate histopathological analysis of the basal forebrain cholinergic nuclei of post-mortem brain tissue in PD and PDD patients and healthy controls. They found: 1) a frank loss of NBM neurons in PDD, 2) $\alpha$-synuclein deposition in the NBM that was significantly higher in PDD versus $P D, 3$ ) a decrease in cholineacetyltransferase (CAT) activity as a marker of cholinergic transmission in the hippocampus in PDD, and 4) frontal neocortical loss of CAT activity in PD and PDD. Cholinergic dysfunction of the frontal cortex is also present in nondemented PD patients without significant structural loss of NBM neurons, and has been attributed to $\alpha$-synuclein deposition in NBM neurons that may decrease neurotransmitter production [21]. The NBM sends out projections to medial temporal structures, the amygdala, the frontoparietal cortex, and temporoparietal association areas [18]. It is the major source of cholinergic transmission to the neocortex, and has been functionally associated with the control of attention and maintenance of arousal, both key functions for appropriate learning and memory formation [5]. Molecular imaging of acetylcholinesterase activity by positron emission tomography (PET) revealed that cholinergic denervation of the cerebral cortex is an early phenomenon in PD and is more widespread and profound in PDD patients [37].

Recently, advanced MRI-based morphometric analysis techniques of the NBM volume have been developed [23], and loss of NBM volume in PD is associated with baseline cognitive deficits $[7,16]$ and longitudinal cognitive decline [34, 36]. Recently, Pereira et al. [33] performed a longitudinal study in patients with PDD or who developed PDD during the study. They reported that atrophy of the NBM precedes and predicts future dementia in PD. Baseline volumes and longitudinal changes in the NBM were associated with worse global cognition and semantic and phonemic fluency abilities. In accordance with the functional deficit of posterior cortical regions, Gang et al. showed an association between reductions in NBM volume and parietal glucose metabolism in PDD [15].

Rivastigmine is the only approved medication addressing cognitive impairment in PDD, and effect sizes in 
clinical studies were relatively small [3]. No diseasemodifying treatments with effects on cognition in PD are currently available. Deep brain stimulation (DBS) is an established, highly-effective treatment option in movement disorders [29], with an evolving spectrum of indications that increasingly includes psychiatric disorders [28]. DBS of the NBM has been used in single cases and small patient cohorts. In contrast to conventional DBS targets, NBM-DBS has some exceptional features as the degenerating nucleus itself is the target for electrode placement [26], requiring low-frequency stimulation to activate axon structures. Low-frequency NBM stimulation in rats led to a marked increase in acetylcholine emission in the neocortex [27]. Comparable animal trials demonstrated amelioration of attention and memory functions after NBM-DBS, and more impaired baseline memory functions led to greater effects $[9,24,30]$.

A first case study published by Freund et al. reported the effects of bilateral NBM-DBS and subthalamic nucleus (STN)-DBS as a standard treatment of motor symptoms in a 71-year-old man with severe PDD [14]. Improvements in short-term memory, attention, concentration, alertness, drive, spontaneity, and preoperative apraxia were reported following NBM-DBS $(20 \mathrm{~Hz})$, which remained stable over 18 months [6]. Gratwicke et al. [19] recently published an exploratory randomized, double-blind, crossover trial of NBM-DBS in six patients with PDD. Electrodes were placed on a trajectory that straddled the internal part of the globus pallidus internus (GPI), providing the potential for subsequent conventional GPI-DBS for coexisting motor impairments. After surgery, patients were assigned to receive active NBM-DBS or sham stimulation for 6 weeks, with crossover for another 6 weeks. The primary outcome was the difference in scores measuring verbal learning, working memory, verbal fluency, attention, and psychomotor speed/reaction times between groups. Surgery and stimulation were well tolerated by all six patients, with no reported serious adverse events. No consistent improvements were observed in primary cognitive outcomes or the resting-state functional magnetic resonance imaging. However, an improvement in Neuropsychiatric Inventory (NPI) scores was observed in two patients with NBM-DBS.

Case reports and small exploratory trials applying NBM-DBS in PD-associated MCI, Alzheimer's disease (AD), and dementia with Lewy bodies (DLB) have also been reported. Nombela et al. described NBM-DBS in a patient with PD-associated MCI [31]. NBM-DBS was combined with GPI-DBS via the same lead, using different contacts and different stimulation frequencies. After 3 months of combined GPI/NBM-DBS, improvements were noted in all neuropsychological measurements apart from semantic verbal fluency and reverse digit span. In the $\mathrm{AD}$ trial, cognition (Mini-Mental State Examination, MMSE) was stable over 12 months after DBS in all 10 patients and correlated with a preserved fronto-parieto-temporal cortical thickness [4]. Gratwicke et al. replicated their randomized, double-blind, crossover trial in six patients with DLB [20]. No consistent improvements were observed in exploratory clinical outcome measures, but the severity of neuropsychiatric symptoms reduced with NBM-DBS in three patients. In all published cases, NBM-DBS proved to be feasible and well tolerated, with only temporary related adverse events.

Thus, initial results in PDD, AD, and DLB demonstrate the safety and feasibility of NBM-DBS and provide an indication of efficacy regarding neuropsychiatric symptoms. However, neurosurgical intervention for implantation of NBM electrodes alone is currently unjustifiable because it would not address the complex motor and non-motor syndrome associated with advanced PD. Therefore, in our study only patients fulfilling established motor indications (motor response fluctuations or dyskinesia) for STN-DBS will be selected [8]. Patients with cognitive impairment are commonly excluded from this therapy due to safety concerns. This exclusion, based on expert opinion and fear of perioperative delirium or cognitive worsening rather than controlled clinical trial results, needs to be critically reconsidered given the increasing evidence regarding the cognitive safety of STN-DBS in non-demented patients and improved surgical techniques. Moreover, STN-DBS allows a marked reduction in dopaminergic medication, now an indication for this therapy in cases of non-motor dopaminergic adverse events such as impulse control disorders or levodopa-dysregulation syndrome, which are difficult to manage pharmacologically [38]. The reduction in dopaminergic medication may also benefit PDD patients, to reduce the risk of delirium or hyperdopaminergic behaviors aggravating PDD-associated symptoms.

\section{Methods}

\section{Aim of the trial}

The only currently-available treatment option in PDD consists of oral rivastigmine, which increases acetylcholine levels in the brain by inhibiting cholinesterase [12]. Its efficacy is limited, especially when compared to the effects of levodopa on motor symptoms. Diseasemodifying treatment strategies are lacking [2]. Following the assumption of initial axonal dysfunction and subsequent neurodegeneration of the NBM, augmentative neurostimulation of the NBM should increase cholinergic output from the basal forebrain and reconstitute neocortical functions. Our study (DEMPARK-DBS) will evaluate the safety of combined bilateral STN-NBM- 
DBS in PDD patients, and will clarify whether additional NBM-DBS improves or slows cognitive decline.

\section{Study description and study design}

This will be a prospective, single center, Phase $1 \mathrm{~b}$ study with a double-blind, randomized, sham-stimulation controlled, delayed activation of NBM-DBS (staggered onset) design. We aim to include 12 patients with PDD fulfilling eligibility criteria listed in Table 1 . Patients must be on stable doses of antiparkinsonian and antidementia medications for at least 4 weeks prior to screening assessment. Medications and dosages may be adjusted as necessary after DBS surgery. Figure 1 shows the study schedule of DEMPARK-DBS.

During the screening visit, the PDD diagnosis will be confirmed by the Movement Disorders Society (MDS) criteria using the algorithm for PDD at level I [11]. The MMSE total score must lie between 10 and 24 points, indicating mild-to-moderately severe dementia. Patients will fulfill clinical inclusion criteria for STN-DBS in terms of motor scores and requirements for local/general anesthesia.

The preoperative baseline examination will be performed 4 weeks prior to the DBS operation. Table 2 shows the full data set to be collected at pre- and postoperative Baseline and Visits 1-3 to achieve exploratory study endpoints, and includes assessment of motor function in the medication-"off" state after 12-h withdrawal of dopaminergic medication and medication-"on" state after standard levodopa challenge; video recording will also be used for documentation. All other scores and scales will be performed in the medication-"on" state, to assess global cognitive function, neuropsychiatric symptoms, and a number of domain-specific tests as part of a detailed neuropsychiatric battery, as well as quality of life, health economics, and caregiver burden. During all study visits, adverse events will be documented and rated for frequency and severity.

All participants will undergo stereotactic implantation of deep brain electrodes. The neurostimulation device used will be the Vercise ${ }^{\mathrm{mat}}$ System (Boston Scientific Corporation (BSC)), consisting of an implantable pulse generator (IPG), integrated rechargeable battery, DBS leads, surgical tools, and external devices (programming system, remote control, and charging system; Table 3). To allow delivery of stimulation pulses to four DBS electrodes with different stimulation frequencies (STN: 100$200 \mathrm{~Hz}$; NBM: $20-80 \mathrm{~Hz}$ ), the 22-cc Precision Spinal Cord Stimulation IPG II splitter (BSC) will be used. The DBS procedure will follow local standard operating procedures on perioperative management and stereotactic procedure. A preoperative MRI-scan under general anesthesia and postoperative image control of electrode positioning will be performed in all patients.

Postsurgical baseline evaluation will be performed at 4( \pm 1$)$ weeks after surgery. Motor assessments will be performed in the medication-"off" state. Subthalamic neurostimulation will be initiated in all patients using individualized stimulation parameters determined via monopolar review.

Twelve weeks after STN activation, neurostimulation Visit 1 (V1) will take place. At this time, baseline evaluation of the clinical global impression of change (ADCSCGIC) will be performed by a blinded rater not involved in medical treatment and with no information about the results of other scales and scores. For the first baseline interview with the patient and caregiver, documented medical history will be the only additional information that may be studied. Motor assessments will be performed in the medication-"off" and -"on" state with

Table 1 Eligibility criteria

Inclusion criteria:
1. Age at enrollment: $35-75$ years.
2. Diagnosis of idiopathic PD with probable PDD, defined by MDS
consensus guidelines [13].
3. Mild-to-moderately severe dementia, defined by MMSE score $10-24$.
4. Duration of bilateral idiopathic PD: $\geq 5$ years of motor symptoms.
5. Severity of bilateral idiopathic PD in medication-off state: modified
Hoehn and Yahr stage $\geq 2$.
6. Unified Parkinson's Disease Rating Scale (UPDRS) III score $\geq 30$ in
medication-off/stimulation-off state.
7. Levodopa must improve PD symptoms by $\geq 30 \%$ in levodopa challenge
test (UPDRS III score).
8. PDD with symptom onset at least 2 years after first symptoms of PD.
9. Willing and able to comply with all visits and study-related procedures
(e.g., using the remote control and charging systems, completing the
motor diary) if mentally competent or, if incompetent, their legally-
authorized representatives.
10. Able to understand the study requirements and treatment procedures
and provide written informed consent before any study-specific tests or
procedures are performed. If mentally incompetent, the legally-authorized
representative will provide written informed consent.

\section{Exclusion criteria:}

1. Any significant psychiatric problems, including acute confusional state (delirium), ongoing psychosis, or clinically significant depression.

2. Any current drug or alcohol abuse.

3. Any history of recurrent or unprovoked seizures.

4. Any prior movement disorder treatments involving intracranial surgery or device implantation.

5. History of neurostimulation intolerance in any area of the body. 6. Any significant medical condition likely to interfere with study procedures or confound evaluation of study endpoints, including any terminal illness with survival $<12$ months.

7. Participation in another drug, device, or biologics trial concurrently or within the preceding 30 days. Any other trial participation should be approved by the Principal Investigators.

8. Pregnancy, breastfeeding, or lack of reliable contraception. 


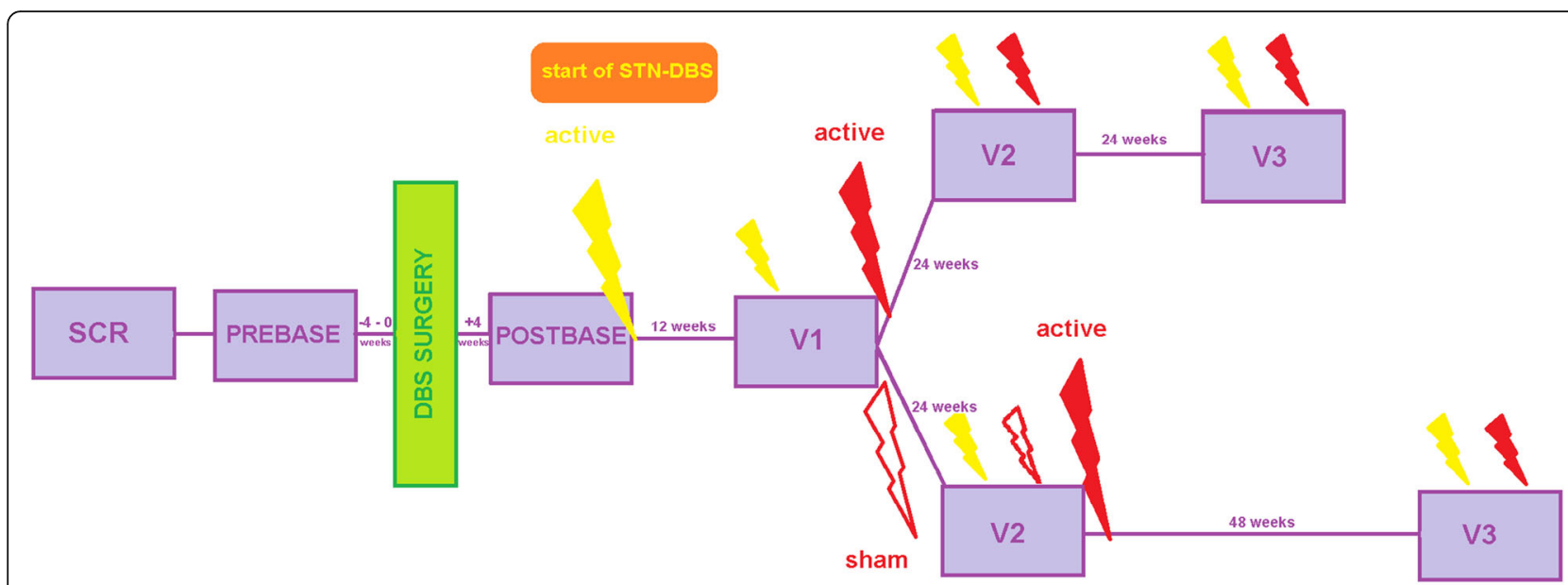

\section{start of NBM-DBS}

Fig. 1 Visit schedule of DEMPARK-DBS: Icon "flash" = 'activation' or 'active' DBS. PREBASE, presurgical baseline visit; POSTBASE, postsurgical baseline visit; SCR, screening visit; V1, Visit 1; V2, Visit 2; V3, Visit 3

active STN-DBS; all other scales and scores will be evaluated in the medication-"on" state with active STN-DBS. Subsequently, medication and STN-DBS parameters will be optimized if necessary, followed by $1: 1$ blinded randomization to active or sham NBM-DBS. After another 24 weeks, Visit 2 (V2) will take place. To evaluate CGIC compared to V1, patient and caregivers will be reinterviewed by the blinded rater that performed the baseline interview. This time, no additional information may be provided from medical records or other information sources. Motor assessments will be performed in the medication-"off" and -"on" state with active STNDBS and active or sham NBM-DBS; all other scales and scores will be evaluated in the medication-"on" state with active STN-DBS and active or sham NBM-DBS. Subsequently, STN-DBS will be adapted if necessary and active NBM-DBS continued in all patients. Visit 3 (V3) will be performed 48 weeks after activation of NBM-DBS

Table 2 Data set: assessment at presurgical/postsurgical baseline, visits 1-3

\begin{tabular}{|c|c|}
\hline Motor assessments & $\begin{array}{l}\text { - Movement Disorder Society-Unified Parkinson's Disease Rating Scale (MDS-UPDRS) III (medication-off/-on } \\
\text { state) } \\
\text { - MDS-UPDRS I, IV } \\
\text { - Clinical Dyskinesia Rating Scale (CDRS) }\end{array}$ \\
\hline Global cognitive function & - Alzheimer's Disease Assessment Scale, cognitive subscale (ADAS-cog) \\
\hline Activities of daily living & $\begin{array}{l}\text { - Alzheimer's Disease Cooperative Study, Activities of Daily Living (ADCS-ADL) } \\
\text { - MDS-UPDRS II }\end{array}$ \\
\hline Neuropsychiatric symptoms & $\begin{array}{l}\text { - Beck Depression Inventory (BDI) II, } \\
\text { - Starkstein Apathy Scale } \\
\text { - Neuropsychiatric inventory (NPI) }\end{array}$ \\
\hline $\begin{array}{l}\text { Domain specific (frontal executive) } \\
\text { tests }\end{array}$ & $\begin{array}{l}\text { - Delis-Kaplan Executive Function Systems (D-KEFS) verbal fluency battery } \\
\text { - Wisconsin Card Sorting Test (modified version) } \\
\text { - Trail Making Task (TMT) Part A + B } \\
\text { - Stroop Test (Victoria Version) } \\
\text { - Symbol Digit Modalities Test (SDMT) }\end{array}$ \\
\hline Domain specific (attention) test & - Brief Test of Attention (BTA) \\
\hline Quality of life & $\begin{array}{l}\text { - Parkinson's Disease Questionnaire (PDQ39) } \\
\text { - EuroQol (EQ)-5d }\end{array}$ \\
\hline Health economics & - EQ-5d/quality-adjusted life years (QALYs) \\
\hline Caregiver burden/quality of life & $\begin{array}{l}\text { - Zarit Burden Interview (ZBI) } \\
\text { - Short form (SF)-36 }\end{array}$ \\
\hline Clinical global impression of change & $\begin{array}{l}\text { - Baseline interview: Visit } 1 \\
\text { - Follow-up: Visits } 2 \text { \& } 3\end{array}$ \\
\hline
\end{tabular}


Table 3 Details of the vercise ${ }^{\mathrm{TM}}$ neuromodulation system (Boston Scientific Corporation (BSC))

Vercise TM IPG Kit Model Nr. DB-1110-C

Vercise TM Lead Kit, 30 cm Model Nr. DB-2201-30-C

$55 \mathrm{~cm} 8$ Contact Extension Kit Model Nr. NM-3138-55

Vercise TM Physician's Spare Kit Model Nr. DB-2500-C

Vercise TM Clinician Programmer (M400) Model Nr. DB-7151-20-C

Tunneling Tool, 35 cm Long Model Nr. SC-4254

Holder, IR Interface Model Nr. NM-4502

Vercise TM Charging Collar Aceesseries Model Nr. DB-6300-C

Vercise TM Remote Control Kit Model Nr. DB-5500-C

Vercise TM Remote Control w/batteries (Ti) Model Nr. DB-5212-C

Vercise TM Charging System Kit Model Nr. DB-6412-EU-C

Charger Model Nr. NM-5312

Base Station Model Nr. NM-5305

D4 Splitter $2 \times 4$ Model Nr. SC-3304-xx

in both patient groups, i.e., 24 weeks after V2 in the NBM-DBS group, 48 weeks after V2 in the sham NBMDBS group. The CGIC will again be rated compared to V1. After V3, the study will end and medication, STNDBS, and NBM-DBS will be adjusted without restrictions according to clinical needs. An annual follow-up visit for up to 5 years after activation of NBM-DBS will be provided to all participants.

\section{Arms and interventions}

Eligible patients who consent to participation and meet all inclusion and exclusion criteria will receive the following settings in a pre-specified randomized order for NBM-DBS at V2: test stimulation at $60 \mu \mathrm{s}$, $20 \mathrm{~Hz}$, and individually-adjusted amplitude (verified using the GuideXT visualization tool (BSC) if necessary) below the threshold of adverse effects; sham stimulation at $0 \mathrm{~V}, 60 \mu \mathrm{s}, 20 \mathrm{~Hz}$. As we do not anticipate any specific clinical effects after NBM-DBS activation, blinding will be secured by documenting NBM-DBS parameters independent from medical records. Randomization and programming of NMB-DBS will be restricted to three members of the study team not involved in other diagnostic procedures.

\section{Outcome measures}

The primary endpoint will be the safety of combined STN-NBM-DBS, determined by spontaneously-reported adverse events. Exploratory endpoints will comprise the change between V2 to V1 concerning the scales and scores of the full data set listed in Table 2 .

\section{Statistical methods}

This study is exploratory to provide necessary data for sample size considerations for a possible subsequent pivotal trial. Sample size considerations are based on research by Emre et al. [12]; they found a $2.1 \pm 8.2$ point improvement on ADAS-cog with rivastigmine treatment compared to a worsening of $0.7 \pm 7.5$ points with placebo after 24 weeks (baseline $23.8 \pm 10.2$ points). Clearly, such small mean differences in combination with comparatively large standard deviations shown by the rivastigmine study will be only be detectable with appropriate power with sample sizes $(2 * 125=250)$ far above that planned for our study. However, we hope to encounter considerably larger effects for NBM-DBS. Within this purely explorative analysis, we will test the null hypothesis of equal mean ADAS-cog change scores from V1 to V2 for both study treatments (STN-DBS + NBM-DBS versus STN-DBS + sham NBM-DBS) by a Type III F-test within an analysis of covariance (ANCOVA), with baseline ADAS-cog as covariate. Assuming a small-tomoderate correlation between baseline ADAS-cog and ADAS-cog change score from V1 to V2, a sample size of $2 * 6=12$ patients ensures a power of $80 \%$ to detect a standardized mean difference of 1.66 as significant deviation from the null hypothesis of equal mean change scores for both treatments at significance level 0.05. This means we will be able to detect only large effects as significant, but that is not the main objective of this pilot study.

Note that because of the small sample size, statistical testing will not be the focus of data analysis. Despite the known robustness of ANCOVA, it may be difficult to detect violations in ANCOVA assumptions. A critical issue comes from possible differences in the sample distributions of baseline variables between the two treatment groups when comparing mean change scores for endpoints. If appropriate mean change scores resp. distributions of change score will be compared without adjustment for baseline variables by the two-sided T-test resp. by the Mann-Whitney test instead of an F-test within ANCOVA.

\section{Perspective}

DEMPARK-DBS will be the first controlled study to evaluate combined STN-NBM-DBS using four electrodes as originally described by Freund et al. [14]. We will assess the feasibility and safety of combined STNNBM-DBS in patients with PDD and will determine whether additional NBM-DBS improves or slows the progression of cognitive decline. Positive results would provide a basic concept for future studies evaluating the efficacy of NBM-DBS in larger PDD cohorts.

Dopaminergic treatment may worsen cognitive functions in advanced PD [25], and has a number of side 
effects that amplify behavioral disorders also primarily caused by dementia. STN-DBS allows a significant reduction in dopaminergic medication; by reducing associated hyperdopaminergic cognitive and behavioral disorders, it could benefit PDD patients. Proof-of-safety of STN-DBS in PDD might also have an impact on the general selection criteria for DBS in patients with advanced $P D$.

\section{Abbreviations}

Ach: Acetylcholine; AChE: Acetylcholinesterase; ADAS-cog: Alzheimer's Disease Assessment Scale, cognitive subscale; ADCS-ADL: Alzheimer's Disease Cooperative Study, Activities of Daily Living; ADCS-CGIC: Alzheimer's Disease Cooperative Study, Clinical Global Impression of Change; ADL: Activities of daily living; BDI: Beck Depression Inventory; BSC: Boston Scientific Corporation; BTA: Brief Test of Attention; CAT: Cholineacetyltransferase; CDRS: Clinical Dyskinesia Rating Scale; DBS: Deep brain stimulation; DKEFS: Delis-Kaplan Executive Function Systems; EQ-5d: EuroQol; MCI: Mild cognitive impairment; MDS: Movement Disorder Society; MMSE: Mini-Mental State Examination; NBM: Nucleus basalis of Meynert; NPI: Neuropsychiatric Inventory; PD: Parkinson's disease; PDD: Parkinson's disease dementia; PDQ: Parkinson's Disease Questionnaire; PET: Positron Emission Tomography; QALY: Quality-adjusted life year; SDMT: Symbol Digit Modalities Test; SF: Short Form (SF-36); STN: Subthalamic nucleus; TMT: Trail Making Task; UPDRS: Unified Parkinson's Disease Rating Scale; ZBI: Zarit Burden Interview

\section{Acknowledgements}

Not applicable.

\section{Authors' contributions}

$J V, C D, C M, P U H$ and UM developed the study design and protocol. PUH and UM in particular were responsible for statistical and biometrical issues. $\mathrm{JV}, C D$ and PC are active clinical investigators in DEMPARK-DBS. CD and JV prepared the first draft of the manuscript, which was revised afterwards by FS, PC, CM, PUH and UM. All authors read and approved the final manuscript.

\section{Funding}

Funding of this study was granted by Boston Scientific Corporation. This institution had no influence on establishing the study design or writing this manuscript. Collection, analysis and interpretation of data will be run independently from the funding bodies.

\section{Availability of data and materials}

Not applicable.

\section{Ethics approval and consent to participate}

The protocol conforms to the principles of the declaration of Helsinki and was approved by the ethic committee of the Medical faculty of the University of Wuerzburg (reference number: 173/15_mm). All patients have to give their written consent on study participation. Patients, who are not able to give their written informed consent, are substituted by their legal representatives. Patients and/or their representatives need to be informed about the study in detailed fashion by their treating physician and must obtain sufficient time for consideration. Specific risks of the particular interventions were explained by the responsible specialists (DBS operation: neurosurgeon, general anesthesia: anesthesiologist).

\section{Consent for publication}

\section{Not applicable.}

\section{Competing interests}

$C D$ and UM report no conflict of interests. FS reports grants and personal fees from Boston Scientific, Abbott and Medtronic. CM received lecture honoraria by Boston Scientific. PC reports consultancy works for Brainlab and ProPhase. He received honoraria from Boehringer Ingelheim, Boston Scientific, Brainlab, Desitin and the DSO. PUH reports research grants from German Ministry of Research and Education, German Research Foundation, European Union, Charité - Universitätsmedizin Berlin, Berlin Chamber of
Physicians, German Parkinson Society, University Hospital Würzburg, Robert Koch Institute, German Heart Foundation, Federal Joint Committee (G-BA) within the Innovationfond, University Hospital Heidelberg (within RASUNOAprime; supported by an unrestricted research grant to the University Hospital Heidelberg from Bayer, BMS, Boehringer-Ingelheim, Daiichi Sankyo), Charité Universitätsmedizin Berlin (within Mondafis; supported by an unrestricted research grant to the Charité from Bayer), University Göttingen (within FIND-AF randomized; supported by an unrestricted research grant to the University Göttingen from Boehringer-Ingelheim), outside the submitted work. JV received consulting and lecture fees from Boston Scientific, Medtronic and Newronika. Research grants from the German Research Foundation, the German Ministry of Research and Education, Boston Scientific and Medtronic. Lecture Honoraria from UCB, Zambon, Abbott.

\section{Author details}

${ }^{1}$ Department of Neurology, University Hospital Würzburg, Josef-Schneider-Str. 11,97080 Würzburg, Germany. ${ }^{2}$ Department of Neurosurgery, University Hospital Würzburg, Josef-Schneider-Str. 11, 97080 Würzburg, Germany. ${ }^{3}$ Clinical Trial Center, University Hospital Würzburg, Josef-Schneider-Str. 2, 97080 Würzburg, Germany. ${ }^{4}$ Institute of Clinical Epidemiology and Biometry, University of Würzburg, Josef-Schneider-Str. 2, 97080 Würzburg, Germany.

Received: 17 July 2020 Accepted: 1 September 2020

Published online: 19 October 2020

\section{References}

1. Aarsland, D., Andersen, K., Larsen, J. P., Lolk, A., \& Kragh-Sørensen, P. (2003). Prevalence and characteristics of dementia in Parkinson disease: an 8-year prospective study. Archives of Neurology, 60(3), 387-392. https://doi.org/10 1001/archneur.60.3.387.

2. Aarsland, D., Beyer, M. K., \& Kurz, M. W. (2008). Dementia in Parkinson's disease. Current Opinion in Neurology, 21(6), 676-682. https://doi.org/10. 1097/WCO.0b013e3283168dfo.

3. Aarsland, D., Creese, B., Politis, M., Chaudhuri, K. R., Ffytche, D. H., Weintraub, D., \& Ballard, C. (2017). Cognitive decline in Parkinson disease. Nature Reviews Neurology, 13(4), 217-231. https://doi.org/10.1038/nrneurol.2017.27.

4. Baldermann, J. C., Hardenacke, K., Hu, X., Köster, P., Horn, A., Freund, H. J., ... Kuhn, J. (2018). Neuroanatomical characteristics associated with response to deep brain stimulation of the nucleus Basalis of Meynert for Alzheimer's disease. Neuromodulation, 21(2), 184-190. https://doi.org/10.1111/ner.12626.

5. Ballinger, E. C., Ananth, M., Talmage, D. A., \& Role, L. W. (2016). Basal forebrain cholinergic circuits and signaling in cognition and cognitive decline. Neuron, 91(6), 1199-1218. https://doi.org/10.1016/j.neuron.2016.09. 006.

6. Barnikol, T. T., Pawelczyk, N. B., Barnikol, U. B., Kuhn, J., Lenartz, D., Sturm, V., Freund, H. J. (2010). Changes in apraxia after deep brain stimulation of the nucleus basalis Meynert in a patient with Parkinson dementia syndrome. Movement Disorders, 25(10), 1519-1520. https://doi.org/10.1002/ mds.23141.

7. Barrett, M. J., Sperling, S. A., Blair, J. C., Freeman, C. S., Flanigan, J. L., Smolkin, M. E., ... Druzgal, T. J. (2019). Lower volume, more impairment: Reduced cholinergic basal forebrain grey matter density is associated with impaired cognition in Parkinson disease. Journal of Neurology, Neurosurgery \& Psychiatry, 90(11), 1251-1256. https://doi.org/10.1136/jnnp-2019-320450.

8. Bronstein, J. M., Tagliati, M., Alterman, R. L., Lozano, A. M., Volkmann, J., Stefani, A., ... DeLong, M. R. (2011). Deep brain stimulation for Parkinson disease: an expert consensus and review of key issues. Archives of Neurology, 68(2), 165. https://doi.org/10.1001/archneurol.2010.260.

9. Butt, A. E., Schultz, J. A., Arnold, L. L., Garman, E. E., George, C. L., \& Garraghty, P. E. (2003). Lesions of the rat nucleus basalis magnocellularis disrupt appetitive-to-aversive transfer learning. Integrative Psychological and Behavioral Science, 38(4), 253-271. https://doi.org/10.1007/BF02688857.

10. Dirnberger, G., \& Jahanshahi, M. (2013). Executive dysfunction in Parkinson's disease: a review. Journal of Neuropsychology, 7(2), 193-224. https://doi.org/ 10.1111/jnp.12028.

11. Dubois, B., Burn, D., Goetz, C., Aarsland, D., Brown, R. G., Broe, G. A.r... Emre, M. (2007). Diagnostic procedures for Parkinson's disease dementia: recommendations from the movement disorder society task force. Movement Disorders, 22(16), 2314-2324. https://doi.org/10.1002/mds.21844.

12. Emre, M., Aarsland, D., Albanese, A., Byrne, E. J., Deuschl, G., De Deyn, P. P., .. Lane, R. (2004). Rivastigmine for dementia associated with Parkinson's 
disease. New England Journal of Medicine, 351(24), 2509-2518. https://doi. org/10.1056/NEJMoa041470.

13. Emre, M., Aarsland, D., Brown, R., Burn, D. J., Duyckaerts, C., Mizuno, Y., ... Dubois, B. (2007). Clinical diagnostic criteria for dementia associated with Parkinson's disease. Movement Disorders, 22(12), 1689-1707; quiz 1837. https://doi.org/10.1002/mds.21507.

14. Freund, H. J., Kuhn, J., Lenartz, D., Mai, J. K., Schnell, T., Klosterkoetter, J., \& Sturm, V. (2009). Cognitive functions in a patient with Parkinson-dementia syndrome undergoing deep brain stimulation. Archives of Neurology, 66(6), 781-785. https://doi.org/10.1001/archneurol.2009.102.

15. Gang, M., Baba, T., Hosokai, Y., Nishio, Y., Kikuchi, A., Hirayama, K., ... Suzuki, K. (2020). Clinical and cerebral metabolic changes in Parkinson's disease with basal forebrain atrophy. Movement Disorders. https://doi.org/10.1002/ mds.27988.

16. Gargouri, F., Gallea, C., Mongin, M., Pyatigorskaya, N., Valabregue, R., Ewenczyk, C., ... Lehéricy, S. (2019). Multimodal magnetic resonance imaging investigation of basal forebrain damage and cognitive deficits in Parkinson's disease. Movement Disorders, 34(4), 516-525. https://doi.org/10. 1002/mds.27561.

17. Gaspar, P., \& Gray, F. (1984). Dementia in idiopathic Parkinson's disease. A neuropathological study of 32 cases. Acta Neuropathologica, 64(1), 43-52. https://doi.org/10.1007/BF00695605.

18. Gratwicke, J., Kahan, J., Zrinzo, L., Hariz, M., Limousin, P., Foltynie, T., \& Jahanshahi, M. (2013). The nucleus basalis of Meynert: a new target for deep brain stimulation in dementia? Neuroscience \& Biobehavioral Reviews, 37(10 Pt 2), 2676-2688. https://doi.org/10.1016/j.neubiorev.2013.09.003.

19. Gratwicke, J., Zrinzo, L., Kahan, J., Peters, A., Beigi, M., Akram, H., ... Foltynie, T. (2018). Bilateral deep brain stimulation of the nucleus Basalis of Meynert for Parkinson disease dementia: a randomized clinical trial. Journal of the American Medical Association Neurology, 75(2), 169-178. https://doi.org/10. 1001/jamaneurol.2017.3762

20. Gratwicke, J., Zrinzo, L., Kahan, J., Peters, A., Brechany, U., McNichol, A., .. Foltynie, T. (2020). Bilateral nucleus basalis of Meynert deep brain stimulation for dementia with Lewy bodies: a randomised clinical trial. Brain Stimulation, 13(4), 1031-1039. https://doi.org/10.1016/j.brs.2020.04.010.

21. Hall, H., Reyes, S., Landeck, N., Bye, C., Leanza, G., Double, K., ... Kirik, D. (2014). Hippocampal Lewy pathology and cholinergic dysfunction are associated with dementia in Parkinson's disease. Brain, 137(Pt 9), 2493-2508. https://doi.org/10.1093/brain/awu193.

22. Kehagia, A. A., Barker, R. A., \& Robbins, T. W. (2010). Neuropsychological and clinical heterogeneity of cognitive impairment and dementia in patients with Parkinson's disease. Lancet Neurology, 9(12), 1200-1213. https://doi.org/ 10.1016/S1474-4422(10)70212-X.

23. Kilimann, I., Grothe, M., Heinsen, H., Alho, E. J., Grinberg, L., Amaro, E., ... Teipel, S. J. (2014). Subregional basal forebrain atrophy in Alzheimer's disease: a multicenter study. Journal of Alzheimers Disease, 40(3), 687-700. https://doi.org/10.3233/JAD-132345.

24. Koulousakis, P., van den Hove, D., Visser-Vandewalle, V., \& Sesia, T. (2020). Cognitive improvements after intermittent deep brain stimulation of the nucleus Basalis of Meynert in a transgenic rat model for Alzheimer's disease: a preliminary approach. Journal of Alzheimers Disease, 73(2), 461-466. https:// doi.org/10.3233/JAD-190919.

25. Kulisevsky, J. (2000). Role of dopamine in learning and memory: implications for the treatment of cognitive dysfunction in patients with Parkinson's disease. Drugs \& Aging, 16(5), 365-379. https://doi.org/10.2165/00002512200016050-00006

26. Kumbhare, D., Palys, V., Toms, J., Wickramasinghe, C. S., Amarasinghe, K., Manic, M., ... Holloway, K. L. (2018). Nucleus Basalis of Meynert stimulation for dementia: theoretical and technical considerations. Frontiers in Neuroscience, 12, 614. https://doi.org/10.3389/fnins.2018.00614.

27. Kurosawa, M., Sato, A., \& Sato, Y. (1989). Stimulation of the nucleus basalis of Meynert increases acetylcholine release in the cerebral cortex in rats. Neuroscience Letters, 98(1), 45-50. https://doi.org/10.1016/03043940(89)90371-6.

28. Lee, D. J., Lozano, C. S., Dallapiazza, R. F., \& Lozano, A. M. (2019). Current and future directions of deep brain stimulation for neurological and psychiatric disorders. Journal of Neurosurgery, 131(2), 333-342. https://doi.org/10.3171/ 2019.4.JNS181761.

29. Limousin, P., \& Foltynie, T. (2019). Long-term outcomes of deep brain stimulation in Parkinson disease. Nature Reviews Neurology, 15(4), 234-242. https://doi.org/10.1038/s41582-019-0145-9.
30. Liu, R., Crawford, J., Callahan, P. M., Terry, A. V., Constantinidis, C., \& Blake, D. T. (2017a). Intermittent stimulation of the nucleus Basalis of Meynert improves working memory in adult monkeys. Current Biology, 27(17), 26402646.e2644. https://doi.org/10.1016/j.cub.2017.07.021.

31. Nombela, C., Lozano, A., Villanueva, C., \& Barcia, J. A. (2019). Simultaneous stimulation of the Globus Pallidus Interna and the nucleus Basalis of Meynert in the Parkinson-Dementia Syndrome. Dementia and Geriatric Cognitive Disorders, 47(1-2), 19-28. https://doi.org/10.1159/000493094.

32. Pagonabarraga, J., Kulisevsky, J., Llebaria, G., García-Sánchez, C., PascualSedano, B., \& Gironell, A. (2008). Parkinson's disease-cognitive rating scale: a new cognitive scale specific for Parkinson's disease. Movement Disorders, 23(7), 998-1005. https://doi.org/10.1002/mds.22007.

33. Pereira, J. B., Hall, S., Jalakas, M., Grothe, M. J., Strandberg, O., Stomrud, E., ... Hansson, O. (2020). Longitudinal degeneration of the basal forebrain predicts subsequent dementia in Parkinson's disease. Neurobiology of Disease, 139, 104831. https://doi.org/10.1016/.nbd.2020.104831.

34. Ray, N. J., Bradburn, S., Murgatroyd, C., Toseeb, U., Mir, P., Kountouriotis, G. K. .. Grothe, M. J. (2018). In vivo cholinergic basal forebrain atrophy predicts cognitive decline in de novo Parkinson's disease. Brain, 141(1), 165-176. https://doi.org/10.1093/brain/awx310.

35. Robbins, T. W., \& Cools, R. (2014). Cognitive deficits in Parkinson's disease: a cognitive neuroscience perspective. Movement Disorders, 29(5), 597-607. https://doi.org/10.1002/mds.25853.

36. Schulz, J., Pagano, G., Fernández Bonfante, J. A., Wilson, H., \& Politis, M. (2018). Nucleus basalis of Meynert degeneration precedes and predicts cognitive impairment in Parkinson's disease. Brain, 141(5), 1501-1516. https://doi.org/10.1093/brain/awy072.

37. Shimada, H., Hirano, S., Shinotoh, H., Aotsuka, A., Sato, K., Tanaka, N., ... Irie, T. (2009). Mapping of brain acetylcholinesterase alterations in Lewy body disease by PET. Neurology, 73(4), 273-278. https://doi.org/10.1212/WNL. 0b013e3181ab2b58.

38. Weintraub, D., David, A. S., Evans, A. H., Grant, J. E., \& Stacy, M. (2015). Clinical spectrum of impulse control disorders in Parkinson's disease. Movement Disorders, 30(2), 121-127. https://doi.org/10.1002/mds.26016.

39. Williams-Gray, C. H., Foltynie, T., Brayne, C. E., Robbins, T. W., \& Barker, R. A. (2007). Evolution of cognitive dysfunction in an incident Parkinson's disease cohort. Brain, 130(Pt 7), 1787-1798. https://doi.org/10.1093/brain/awm111.

40. Williams-Gray, C. H., Mason, S. L., Evans, J. R., Foltynie, T., Brayne, C., Robbins, T. W., \& Barker, R. A. (2013). The CamPalGN study of Parkinson's disease: 10year outlook in an incident population-based cohort. Journal of Neurology, Neurosurgery \& Psychiatry, 84(11), 1258-1264. https://doi.org/10.1136/jnnp2013-305277.

\section{Publisher's Note}

Springer Nature remains neutral with regard to jurisdictional claims in published maps and institutional affiliations.

Ready to submit your research? Choose BMC and benefit from:

- fast, convenient online submission

- thorough peer review by experienced researchers in your field

- rapid publication on acceptance

- support for research data, including large and complex data types

- gold Open Access which fosters wider collaboration and increased citations

- maximum visibility for your research: over $100 \mathrm{M}$ website views per year

At $\mathrm{BMC}$, research is always in progress.

Learn more biomedcentral.com/submissions 